\title{
Pseudomonas mRNA 2.0: Boosting Gene Expression Through Enhanced mRNA Stability and Translational Efficiency
}

\author{
Dário Neves ${ }^{1}$, Stefan $\operatorname{Vos}^{1}$, Lars M. Blank ${ }^{1}$ and Birgitta E. Ebert ${ }^{1,2,3 *}$ \\ ${ }^{1}$ Institute of Applied Microbiology (iAMB), Aachen Biology and Biotechnology (ABBt), RWTH Aachen University, Aachen, \\ Germany, ${ }^{2}$ Australian Institute for Bioengineering and Nanotechnology, The University of Queensland, Brisbane, QLD, \\ Australia, ${ }^{3}$ CSIRO Synthetic Biology Future Science Platform, Commonwealth Scientific and Industrial Research Organisation \\ (CSIRO), Brisbane, QLD, Australia
}

OPEN ACCESS

Edited by:

Dong-Yup Lee,

College of Engineering,

Sungkyunkwan University,

South Korea

Reviewed by:

Sang Woo Seo,

Seoul National University, South Korea

Pablo Ivan Nikel,

Novo Nordisk Foundation Center for

Biosustainability (DTU

Biosustain), Denmark

Byung-Kwan Cho,

Korea Advanced Institute of Science \&

Technology (KAIST), South Korea

*Correspondence:

Birgitta E. Ebert

birgitta.eber@uq.edu.au

Specialty section:

This article was submitted to

Synthetic Biology,

a section of the journal

Frontiers in Bioengineering and

Biotechnology

Received: 29 September 2019 Accepted: 19 December 2019

Published: 24 January 2020

Citation:

Neves $D$, Vos S, Blank LM and Ebert BE (2020) Pseudomonas mRNA

2.0: Boosting Gene Expression

Through Enhanced mRNA Stability

and Translational Efficiency.

Front. Bioeng. Biotechnol. 7:458.

doi: 10.3389/fbioe.2019.00458
High gene expression of enzymes partaking in recombinant production pathways is a desirable trait among cell factories belonging to all different kingdoms of life. High enzyme abundance is generally aimed for by utilizing strong promoters, which ramp up gene transcription and mRNA levels. Increased protein abundance can alternatively be achieved by optimizing the expression on the post-transcriptional level. Here, we evaluated protein synthesis with a previously proposed optimized gene expression architecture, in which mRNA stability and translation initiation are modulated by genetic parts such as self-cleaving ribozymes and a bicistronic design, which have initially been described to support the standardization of gene expression. The optimized gene expression architecture was tested in Pseudomonas taiwanensis VLB120, a promising, novel microbial cell factory. The expression cassette was employed on a plasmid basis and after single genomic integration. We used three constitutive and two inducible promoters to drive the expression of two fluorescent reporter proteins and a short acetoin biosynthesis pathway. The performance was confronted with that of a traditional expression cassette harboring the same promoter and gene of interest but lacking the genetic parts for increased expression efficiency. The optimized expression cassette granted higher protein abundance independently of the expression basis or promoter used proving its value for applications requiring high protein abundance.

Keywords: synthetic biology, ribozymes, bicistronic design, Pseudomonas taiwanensis VLB120, mRNA stability, high gene expression

\section{INTRODUCTION}

Cell factories have become an established role player in the sustainable production of chemicals and biological products proven with hundreds of billions of USD/year value on global markets (Davy et al., 2017). A commonality in the development of such cell factories is the continuous pursuit of increased productivities through directed or selection-based genetic engineering methods. With both approaches, increasing activity of the partaking pathways commonly leads to the desired rise in productivity. High enzyme activity can be achieved by optimization of transcription, translation, post-translational modifications, and the process conditions (Liu et al., 2013). A common strategy is to employ strong promoters to overexpress product biosynthesis genes. Highly-active promoters 
achieve increased protein production rates by increasing the respective mRNA levels in the cell. However, previous studies have shown that high, recombinant gene expression leads to metabolic burden and consequently to growth impairment (Carneiro et al., 2013; Borkowski et al., 2016). Such hindrances are related to the drainage of biosynthetic precursors, such as nucleotides, or seizing of the cellular transcriptional machinery.

In recent years, Synthetic Biology parts emerged that support high enzyme activities without the need for strong gene expression, thereby contributing to diminishing competition and depletion of the cellular mRNA pool and lightening the metabolic burden. Two translation-focused approaches can be distinguished that target to optimize translation rather than transcription. To this end, the first approach attempts to stabilize mRNA, whereas the second seeks to increase translational efficiency. Increasing mRNA stability is possible by placing stabilizing sequences in the $5^{\prime}$ untranslated region (UTR) that avoid endoribonuclease attacks through their secondary structures as in Escherichia coli (Carrier and Keasling, 1997). The implementation of ribozymes upstream of the ribosome binding site (RBS) allows the insulation of the desired expression cassette from the genetic context (Lou et al., 2012). Besides the intrinsic cleaving activity, the ribozymes developed by Lou et al. contained a 23 nucleotide hairpin downstream of the catalytic core, which additionally adds an mRNA stabilizing trait to this genetic part (Clifton et al., 2018).

One approach within the second category, which focuses on translational efficiency, allows increased expression levels by facilitating the access of the ribosome to the RBS. In the traditional operon architecture (Figure 1A), it is possible that gene of interest (GOI)-dependent secondary structures arise. This folding of the mRNA can block the access of ribosomes to the RBS thereby compromising the desired gene expression (Salis et al., 2009). Mutalik et al. (2013) developed a "bicistronic design" which takes advantage of the intrinsic helicase activity of ribosomes to unveil any RBS-GOI dependent secondary structure and consequently achieve GOI-independent expression. In the bicistronic design, a short leading peptide cistron is allocated upstream of the GOI. The RBS of the GOI is enclosed within the coding sequence of the leading cistron, whereas the start codon of the GOI is fused to the stop codon of the leading cistron. The leading RBS-small peptide combination is known to avoid the formation of secondary structures, which assures the binding of a ribosome. Once the ribosome binds to the first RBS and starts to translate the leading peptide, any possible downstream RBSGOI dependent secondary structures are unveiled by its intrinsic helicase activity exposing the RBS of the GOI. Otto et al. recently combined the bicistronic design with an upstream ribozyme to increase the translational efficiency of heterologous genes integrated in rRNA operons. Here the intention of the ribozyme integration was not to stabilize the mRNA but to increase translation efficiency by reducing a potential steric hindrance by the bulky $5^{\prime} 16 \mathrm{~S}$ mRNA flank and thereby facilitating ribosome docking. To this end, the ribozyme was placed upstream of the RBS, and this integration indeed resulted in a substantial increase in protein production (Otto et al., 2019). Nielsen et al. proposed the compilation of these and further genetic parts

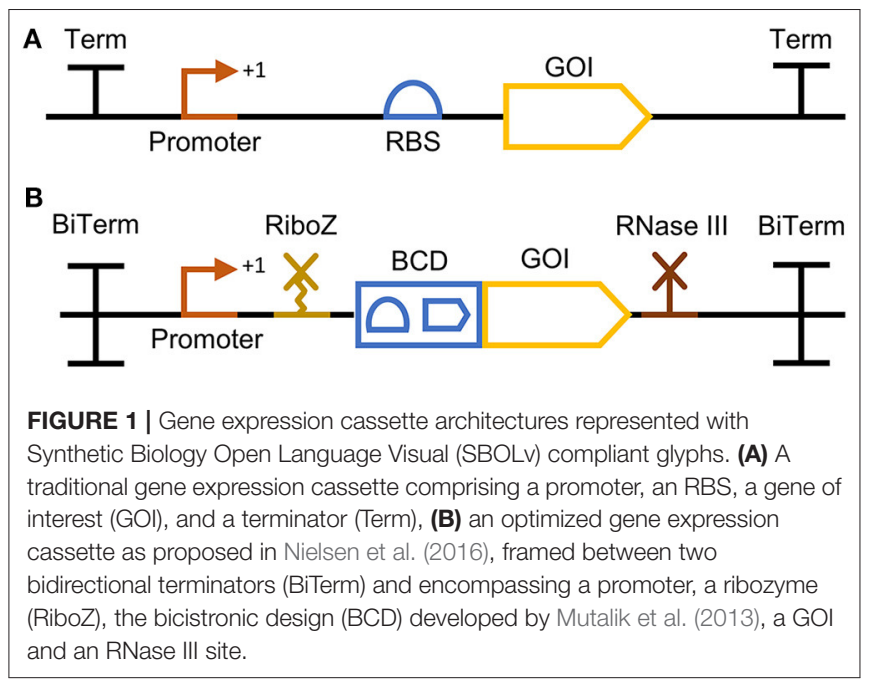

into an overall standardized gene expression cassette for the assembly of genetic circuits (Figure 1B) (Nielsen et al., 2013). Besides the incorporation of the gene expression parts described above, Nielsen et al. proposed the isolation of the expression cassette with bidirectional terminators on both ends and the integration of an RNase III site downstream of the GOI to further reduce context-specific effects. The impact of an RNase III site downstream of the GOI was evaluated by Cambray et al. within the scope of reliable terminator characterization (Cambray et al., 2013). These promoter-independent gene expression tools have been individually characterized but, to our knowledge, a possible synergistic and expression enhancing effect of their combination is yet to be explored.

In this work we constructed optimized gene expression cassettes based on the architecture proposed by Nielsen et al. (2013). and evaluated the performance against traditional configurations using two fluorescence proteins (msfGFP and mCherry) and recombinant acetoin production as readout (Landgraf, 2012). The constructed, optimized gene expression cassettes were evaluated on a plasmid basis and after singlecopy genomic integration. Overall, the traditional and optimized gene expression cassette variants were characterized with three constitutive and two inducible promoters. Reducing the overall size of the optimized gene expression cassette while maintaining its performance was also targeted in this work. Besides the characterization of several constructs, qPCR analysis was performed to elucidate the role of mRNA stability in altered protein expression.

We chose Pseudomonas taiwanensis VLB120 as expression host as this Gram-negative bacterium exhibits industrial relevant metabolic capabilities such as broad carbon source utilization, the ability to proliferate in the presence of organic solvents, and an almost byproduct free metabolism (Köhler et al., 2013). $P$. taiwanensis VLB120 has been proven a suitable biocatalyst for the production of $(S)$-styrene oxide, phenol, isobutyric acid, and 4-hydroxybenzoic acid (Lang et al., 2014; Wynands et al., 
2018; Lenzen et al., 2019). The novel expression device developed in this study contributes to more effective engineering of this emergent and promising biocatalysts and other prokaryotic cell factories.

\section{MATERIALS AND METHODS}

\section{Media and Growth Conditions}

Liquid cultures were grown in a horizontal rotary shaker with a shaking frequency of $200 \mathrm{rpm}$ and a throw of $50 \mathrm{~mm}$ in LB medium or LB medium supplemented with $5 \mathrm{~g} / \mathrm{L}$ glucose and buffered with $11.64 \mathrm{~g} / \mathrm{L} \mathrm{K}_{2} \mathrm{HPO}_{4}$ and $4.89 \mathrm{~g} / \mathrm{L} \mathrm{NaH}_{2} \mathrm{PO}_{4}\left(\mathrm{LB}_{\bmod }\right)$. Pseudomonas strains were grown at $30^{\circ} \mathrm{C}$, whereas E. coli was grown at $37^{\circ} \mathrm{C}$. Solid LB was prepared by adding $1.5 \%(\mathrm{w} / \mathrm{v})$ agar to the medium. Antibiotics were supplemented to the medium for plasmid maintenance and selection purposes. Kanamycin sulfate was added at a concentration of $50 \mathrm{mg} / \mathrm{L}$ for Pseudomonas and E. coli. Gentamycin was used at a concentration of $25 \mathrm{mg} / \mathrm{L}$ for both species. Tetracycline was added only to solid media at a concentration of $30 \mathrm{mg} / \mathrm{L}$ for Pseudomonas and $10 \mathrm{mg} / \mathrm{L}$ for $E$. coli. To induce the pTN1 derived plasmids harboring the nagR/ $\mathrm{P}_{\text {nagAa }}$ promoter system controlling the expression of the fluorescent proteins or the acetoin pathway, 0.01 or $1 \mathrm{mM}$ of sodium salicylate was added, respectively. The inducer isopropyl$\beta$-D-1 thiogalactopyranoside (IPTG) was used at a concentration of $1 \mathrm{mM}$ to induce the $\mathrm{P}_{\text {trc }}$ controlled constructs integrated into the at $\operatorname{Tn} 7$ site of Pseudomonas strains.

The acetoin producing strains were cultivated in airtight $500 \mathrm{~mL}$ serum flasks containing $50 \mathrm{~mL}$ of $\mathrm{LB}_{\text {mod }}$ supplemented with gentamycin (see above). The main cultures were inoculated from an overnight pre-culture to an $\mathrm{OD}_{600}$ of 0.1 . The plasmidbased acetoin pathway genes were induced with sodium salicylate once the cultures reached an $\mathrm{OD}_{600}$ of 1 . Afterwards, samples were collected for HPLC analysis.

The chemicals used were purchased from Merck (Darmstadt, Germany), Sigma-Aldrich (St. Louis, MO, USA), or Carl Roth (Karlsruhe, Germany) unless stated otherwise. Pharmaceutical grade glycerol was kindly provided by Bioeton (Kyritz, Germany).

\section{Plasmid and Strain Construction}

All plasmids were constructed through Gibson assembly (Gibson et al., 2009) using the NEBuilder HiFi DNA Assembly kit (New England Biolabs, Ipswich, MA, USA). Primers used in this study were purchased from Eurofins Genomics (Ebersberg, Germany) as unmodified DNA oligonucleotides. PCR amplification of DNA for cloning purposes was performed using the Q5 High-Fidelity Polymerase (New England Biolabs, Ipswich, MA, USA). All primers and plasmids are listed in the Supplementary Table 1 . The genes ilvB (from E. coli K-12 MG1655, Uniprot P08142, with C83S mutation for improved $\mathrm{k}_{\mathrm{cat}} / \mathrm{K}_{\mathrm{m}}$ ) and aldB (from B. brevis, Uniprot P23616) were codonoptimized for $P$. taiwanensis VLB120 using the online tool OPTIMIZER (Puigbò et al., 2007). Settings were as follows: genetic code, eubacterial; method, guided random; undesired restriction sites were manually excluded, and rare codons with $<6 \%$ usage were avoided by manipulating the input codon usage table. Both codon-optimized genes and their corresponding optimized gene expression parts were ordered as synthetic DNA fragments from Thermo Fisher Scientific; the sequences can be found in the Supplementary Figures 1, 2. The assembled plasmids were transformed into either $\mathrm{NEB}^{\circledR}$ 5-alpha chemically competent E. coli (New England Biolabs, Ipswich, MA, USA) or One Shot ${ }^{\mathrm{TM}}$ PIR2 Chemically Competent E. coli (Thermo Fisher Scientific) cells through heat shock according to the supplier's protocol. Assembled plasmids were transformed into P. taiwanensis VLB120 by electroporation using a GenePulser Xcell (BioRad, Hercules, CA, USA) (settings: $2 \mathrm{~mm}$ cuvette gap, $2.5 \mathrm{kV}, 200 \Omega, 25 \mu \mathrm{F}$ ). For DNA integration into the att $\operatorname{Tn} 7$ locus, the mini-Tn7 delivery vector backbone developed by Zobel et al. (2015) was used and deployed through mating procedures. For mating events, the E. coli donor harboring the mini-Tn7 vector with the constructs to be integrated, the helper strain E. coli HB101 pRK2013, the E. coli DH5 $\lambda$ pir expressing the transpose operon $\operatorname{tn} A B C D$ and the recipient strain were streaked on top of each other on a LB agar plate and incubated at $30^{\circ} \mathrm{C}$ for $12-24 \mathrm{~h}$. Further on, cell material was taken from the bacterial lawn, resuspended in $0.9 \%(\mathrm{w} / \mathrm{v})$ sodium chloride solution and plated on selective cetrimide agar plates. E. coli and Pseudomonas transformants were screened through colony PCR using the OneTaq $2 \times$ Master Mix with standard buffer after lysing colony cell material in alkaline polyethylene glycol, as described by Chomczynski and Rymaszewski (2006). Successful plasmid constructions and genome integrations were confirmed by Sanger sequencing performed by Eurofins Genomics. All strains used in this work are shown in Table $\mathbf{1}$.

\section{Gravimetric Cell Dry Weight Determination}

Overnight cultures of $P$. taiwanensis VLB120 were diluted to pre-established optical densities and filtered through membrane filters with a pore size of $0.2 \mu \mathrm{M}$ which were previously weighted $\left(\mathrm{w}_{0}\right)$ after being dried in a microwave for $3 \mathrm{~min}$ at $350 \mathrm{~W}$. The filter cake was washed three times with distilled water, dried in the microwave for $8 \mathrm{~min}$ at $350 \mathrm{~W}$ and cooled down in a desiccator before weighing $\left(\mathrm{w}_{1}\right)$. The cell dry weight (CDW) was calculated by subtracting $\mathrm{w}_{0}$ from $\mathrm{w}_{1}$ and divided by the volume of the filtered suspensions. One hundred and ninety microliters of the same cell suspensions were transferred to a 96-well microtiter plate (Greiner Bio One), and scattered light signals were recorded at $620 \mathrm{~nm}$ with a gain of 30. Linear regression between the CDW and scattered light signals of the pre-established cell suspensions retrieved the conversion factor between these two units.

\section{Fluorescent Measurements}

All Pseudomonas strains expressing fluorescent reporter proteins were characterized in the microbioreactor system BioLector (m2p-labs, Baesweiler, Germany). Cultivations were performed at $30^{\circ} \mathrm{C}$ with a shaking frequency of $900 \mathrm{rpm}$ and $85 \%$ humidity in a 96-well microtiter plate (Greiner Bio One) containing $190 \mu \mathrm{L}$ of LB media supplemented with required antibiotics for plasmid maintenance and sealed with evaporation reducing foil (Greiner Bio One). The main cultures were inoculated 
TABLE 1 | Strains used in this study.

\begin{tabular}{|c|c|c|}
\hline Strain & Description & References \\
\hline \multicolumn{3}{|l|}{ E. coli } \\
\hline DH5a & $\begin{array}{l}\text { supE44, } \Delta \text { lacU169 ( } \phi 80 l a c Z \Delta M 15), \text { hsdR17 (rK-mK +), recA1, endA1, gyrA96, } \\
\text { thi-1, relA1 }\end{array}$ & Hanahan, 1985 \\
\hline PIR2 & F - $\Delta$ lac169 rpoS(Am) robA1 creC510 hsdR514 endA recA1 uidA(AMlul)::pir & Thermo scientific \\
\hline HB101 pRK2013 & $\mathrm{Sm}^{\mathrm{R}}$, hsdR-M+, proA2, leuB6, thi-1, recA; bears plasmid pRK2013 & Ditta et al., 1980 \\
\hline DH5a pSW-2 & $\mathrm{DH} 5 \alpha$ bearing pSW-2 & Martinez-Garcia and de Lorenzo, 2011 \\
\hline DH5aגpir pTNS1 & DH5 $\alpha \lambda$ pir bearing plasmid pTNS1 & Martinez-Garcia and de Lorenzo, 2011 \\
\hline \multicolumn{3}{|l|}{ P. taiwanensis } \\
\hline VLB120 & Wild type & Panke et al., 1998 \\
\hline VLB120 pTN1_35_T_G & Bearing plasmid pTN1_Syn35_Tra_GFP & This study \\
\hline VLB120 pTN1_35_O_G & Bearing plasmid pTN1_Syn35_Opt_GFP & This study \\
\hline VLB120 pTN1_35_T_C & Bearing plasmid pTN1_Syn35_Tra_mCherry & This study \\
\hline VLB120 pTN1_35_O_C & Bearing plasmid pTN1_Syn35_Opt_mCherry & This study \\
\hline VLB120 pTN1_42_T_G & Bearing plasmid pTN1_Syn42_Tra_GFP & This study \\
\hline VLB120 pTN1_42_O_G & Bearing plasmid pTN1_Syn42_Opt_GFP & This study \\
\hline VLB120 pTN1_42_T_C & Bearing plasmid pTN1_Syn42_Tra_mCherry & This study \\
\hline VLB120 pTN1_42_O_C & Bearing plasmid pTN1_Syn42_Opt_mCherry & This study \\
\hline VLB120 pTN1_75_T_G & Bearing plasmid pTN1_SPA75_Tra_GFP & This study \\
\hline VLB120 pTN1_75_O_G & Bearing plasmid pTN1_SPA75_Opt_GFP & This study \\
\hline VLB120 pTN1_75_T_C & Bearing plasmid pTN1_SPA75_Tra_mCherry & This study \\
\hline VLB120 pTN1_75_O_C & Bearing plasmid pTN1_SPA75_Opt_mCherry & This study \\
\hline VLB120 pTN1_nagR_T_G & Bearing plasmid pTN1_nagR/P nagAa_Tra_GFP & This study \\
\hline VLB120 pTN1_nagR_O_G & Bearing plasmid pTN1_nagR/PnagAa_Opt_GFP & This study \\
\hline VLB120 pTN1_nagR_T_C & Bearing plasmid pTN1_nagR/P ${ }_{\text {nagAa_Tra_mCherry }}$ & This study \\
\hline VLB120 pTN1_nagR_O_C & Bearing plasmid pTN1_nagR/PnagAa_Opt_mCherry & This study \\
\hline VLB120 attTn7::trc_T_GFP & attTn7::tetA_Ptrc_Tra_GFP & This study \\
\hline VLB120 attTn7::trc_O_GFP & attTn7::tetA_Ptrc_Opt_GFP & This study \\
\hline VLB120 pTN1_nagR_T_acetoin & Bearing plasmid pTN1_nagR/PnagAa_Tra_ilvB_aldB & This study \\
\hline VLB120 pTN1_nagR_O_acetoin & Bearing plasmid pTN1_nagR/PnagAa_Opt_ilvB_aldB & This study \\
\hline
\end{tabular}

from an overnight pre-culture to an $\mathrm{OD}_{600}$ of 0.1 . Growth was measured through scattered light signal at $620 \mathrm{~nm}$ with a gain of 30, msfGFP fluorescence was excited at $485 \mathrm{~nm}$ and emission was measured at $520 \mathrm{~nm}$. The measurements were performed with gains of 50 and 70 due to signal overflow of the stronger constructs. mCherry fluorescence was excited at $580 \mathrm{~nm}$, and emission was measured at $610 \mathrm{~nm}$ with a gain of 100. Scattered light values were converted into cell dry weight concentrations with a predetermined calibration curve. Inducer was added to the cultures during the early exponential growth phase. To allow comparison of fluorescence values recorded with different gains, we adapted a published protocol (Beal et al., 2018 ) to convert the msfGFP signals $\mu \mathrm{M}$ units of fluorescein (MFE, $\mu$ moles of fluorescein equivalents). We utilized the Fluorescein NIST-traceable standard (Molecular Probes Inc., Eugene, USA), a calibrated $50 \mu \mathrm{M}$ solution of fluorescein in $100 \mathrm{mM}$ borate buffer (see Supplementary Figure 3 for the calibration curves). This molecular probe exhibits its highest fluorescence at $\mathrm{pH}$ 9.0. Dilution series of the fluorescein standard were performed with $100 \mathrm{mM}$ sodium borate buffer to maintain the $\mathrm{pH}$ above 9 since below this value fluorescein can exist in multiple ionization states which can interfere with calibration.
The specific fluorescence intensity was retrieved from the linear coefficient between the fluorescence signal and CDW and used to characterize the expression constructs. Biological triplicates were performed, and errors presented as the standard deviation of the mean.

\section{qPCR Assays for mRNA Stability Assessment}

Biological triplicates from $P$. taiwanensis VLB120 harboring either the plasmid pTN1_75_T_G or pTN1_75_O_G were grown overnight in $\mathrm{LB}_{\text {mod }}$ supplemented with $25 \mathrm{mg} / \mathrm{mL}$ gentamycin from a glycerol stock. On the following day, the main cultures with the same medium were inoculated to an initial $\mathrm{OD}_{600}$ of 0.1 . Once the cultures reached an $\mathrm{OD}$ of $1,1 \mathrm{mg} / \mathrm{mL}$ rifampicin and $40 \mu \mathrm{g} / \mathrm{mL}$ nalidixic acid were added simultaneously to cease DNA replication and transcription, respectively. Two milliliters of samples were retrieved, centrifuged, and cell pellets flash-frozen in liquid nitrogen and stored at $-80^{\circ} \mathrm{C}$ until further sample treatment. Cell pellets were suspended in $800 \mu \mathrm{L}$ of DNA/RNA protecting buffer from the Monarch Total RNA MiniPrep kit (New England Biolabs, Ipswich, MA, 
USA), transferred into the ZR S6012-50 Bashing beads lysis tubes (Zymo Research, Irvine, CA, USA), and mechanically disrupted for $1 \mathrm{~min}$. The lysate was transferred into a fresh reaction tube and centrifuged for $2 \mathrm{~min}$ at $13,000 \mathrm{rpm}$. The supernatant was transferred into a fresh tube, and the protocol proceeded as described in the Monarch Total RNA MiniPrep kit. qPCR experiments with msfGFP and $r p o B$ primers pairs were performed with $1 \mu \mathrm{L}$ of each RNA sample to confirm that the samples were not contaminated with either genomic or plasmid DNA. Eighty nanograms of RNA of each sample was converted into cDNA using the LunaScript RT SuperMix kit (New England Biolabs, Ipswich, MA, USA). qPCR experiments were performed with the Luna Universal qPCR Master Mix (New England Biolabs, Ipswich, MA, USA). Primer pairs efficiencies for the housekeeping gene $r p o B$ and target gene $m s f G F P$ can be seen in Supplementary Figures 4, 5. qPCR of samples was performed with $1 \mu \mathrm{L}$ of the reverse transcription reaction mixtures. The qPCR was performed with the CFX96 Real-Time PCR Detection System (Biorad, Hercules, CA, USA). qPCR reactions were performed in technical triplicates. Absolute amounts of mRNA transcripts of $m s f G F P$ and $r p o B$ were quantified using the linear calibration curves used for primer pairs efficiencies, which were constructed with either the plasmid harboring the traditional msfGFP expression cassette under the control of the SPA75 or genomic DNA, respectively. The data was analyzed with the Bio-Rad CFX Manager and Microsoft Excel software.

As we normalized the $m s f G F P$ mRNA abundance data with the transcript abundance of the housekeeping gene $r p o B$, the determination of an absolute decay rate for the msfGFP mRNA was not possible. We calculated the delta between the decay rates of the msfGFP and $r p o B$ mRNA instead. Assuming a first-order degradation kinetic for the mRNA of both genes, the time profile of the normalized mRNA data can be described with Equation 1.

$$
\begin{aligned}
& \frac{m R N A_{m s f G F P}(t)}{m R N A_{r p o B}(t)}=\frac{m R N A_{m s f G F P, 0} \cdot \exp ^{k_{m s f G F P} \cdot t}}{m R N A_{r p o B, 0} \cdot \exp ^{k_{r p o B} \cdot t}} \\
& =\frac{m R N A_{m s f G F P, 0}}{m R N A_{r p o B, 0}} \cdot \exp ^{\left(k_{r p o B}-k_{m s f G F P}\right) \cdot t}
\end{aligned}
$$

A non-linear least square algorithm was used in Matlab (The MathWorks Inc., Natick, MA, USA) to fit the experimental data to Equation (1) and to determine the difference in the decay rates of the $r p o B$ and the msfGFP gene.

\section{Analytical Methods}

The samples taken during the acetoin producing cultivations were centrifuged at 13,000 rpm for $1 \mathrm{~min}$, and the supernatant was stored at $-20^{\circ} \mathrm{C}$ until further analysis. To follow the consumption of glucose and production of acetoin, a Beckman System Gold 126 Solvent Module with an organic acid resin column (Polystyrene divinylbenzene copolymer (PS DVB), 300 $\times 8.0 \mathrm{~mm}$, CS-Chromatographie) was used with $5 \mathrm{mM} \mathrm{H}_{2} \mathrm{SO}_{4}$ as eluent at a flow of $0.6 \mathrm{~mL} \mathrm{~h}^{-1}$ for $30 \mathrm{~min}$ at $30^{\circ} \mathrm{C}$. Detection was realized with a System Gold 166 UV detector (Beckman Coulter) and a Smartline RI Detector 2300 (KNAUER Wissenschaftliche Geräte, Berlin, Germany).

\section{RESULTS AND DISCUSSION}

\section{Characterization of Plasmid-Based, Constitutive Fluorescent Protein Expression}

The optimal gene expression profile depends on the specific application. Generally, the use of robust, constitutive promoters is prioritized over inducible promoters for large-scale production as they render the addition of inducers unnecessary and therefore contribute to the cost efficiency of microbial fermentations.

To evaluate the impact and applicability of the consolidated, optimized expression architecture on this type of promoters, we selected two synthetic promoters, Syn42 and Syn35 (originally referred to as BG42 and BG35, respectively), created by Zobel et al. (2015), whereas the third one, SPA75, was obtained from a synthetic promoter library created by Neves et al. (manuscript in preparation). The promoters Syn 42 and SPA75 possess a similar high expression strength in P. taiwanensis VLB120, while the promoter Syn35 exhibits around 25\% of the expression strength of Syn 42 .

These promoters were encompassed within the optimized and traditional gene expression cassette in the pTN1 plasmid backbone, a vector used by the Pseudomonas scientific community (Figure 2A) (Wierckx et al., 2005; Verhoef et al., 2009; Schmitz et al., 2015). The optimized gene expression cassette was framed between two bidirectional terminators to uncouple transcription from its genetic context. For this purpose, two bidirectional terminators characterized by Chen et al., ECK120026481 and ECK120011170, were selected to insulate, respectively, the $5^{\prime}$ and $3^{\prime}$ end of the optimized gene expression cassette (Chen et al., 2013). The ribozyme RiboJ, characterized by Lou et al., and the bicistronic design BCD2, developed by Mutalik et al., were placed downstream of the selected promoters (Lou et al., 2012; Mutalik et al., 2013). The last genetic part included was the RNase III site R1.1, characterized by Cambray et al., and placed downstream of the GOI (Cambray et al., 2013). The traditional versions of the gene expression cassettes were obtained by omitting the enhancing genetic parts and including the $2^{\text {nd }}$ RBS of the BCD2 to maintain the ribosome affinity toward the mRNA between the two expression systems (Figure 2A). It has been demonstrated that certain combinations of RBS and GOI result in secondary structures, which inhibit translation (Mutalik et al., 2013). The occurrence of such secondary structures in the tested traditional constructs cannot be excluded, and a contribution from the BCD to increased expression levels due to the abolishment of these structures should be considered.

We evaluated the 12 constructs in microtiter plate cultivations with online measurements of fluorescence and scattered light. The scattered light values were converted into cell dry weight units, whereas the arbitrary $m s f G F P$ fluorescence units were transformed into equivalents of fluorescein (MFE) to allow a direct comparison between experiments ran with different measurement settings. The mCherry fluorescence values were not converted since all experiments were performed with the same settings. However, we propose the broad implementation of such standardized fluorescence units to facilitate results comparison 


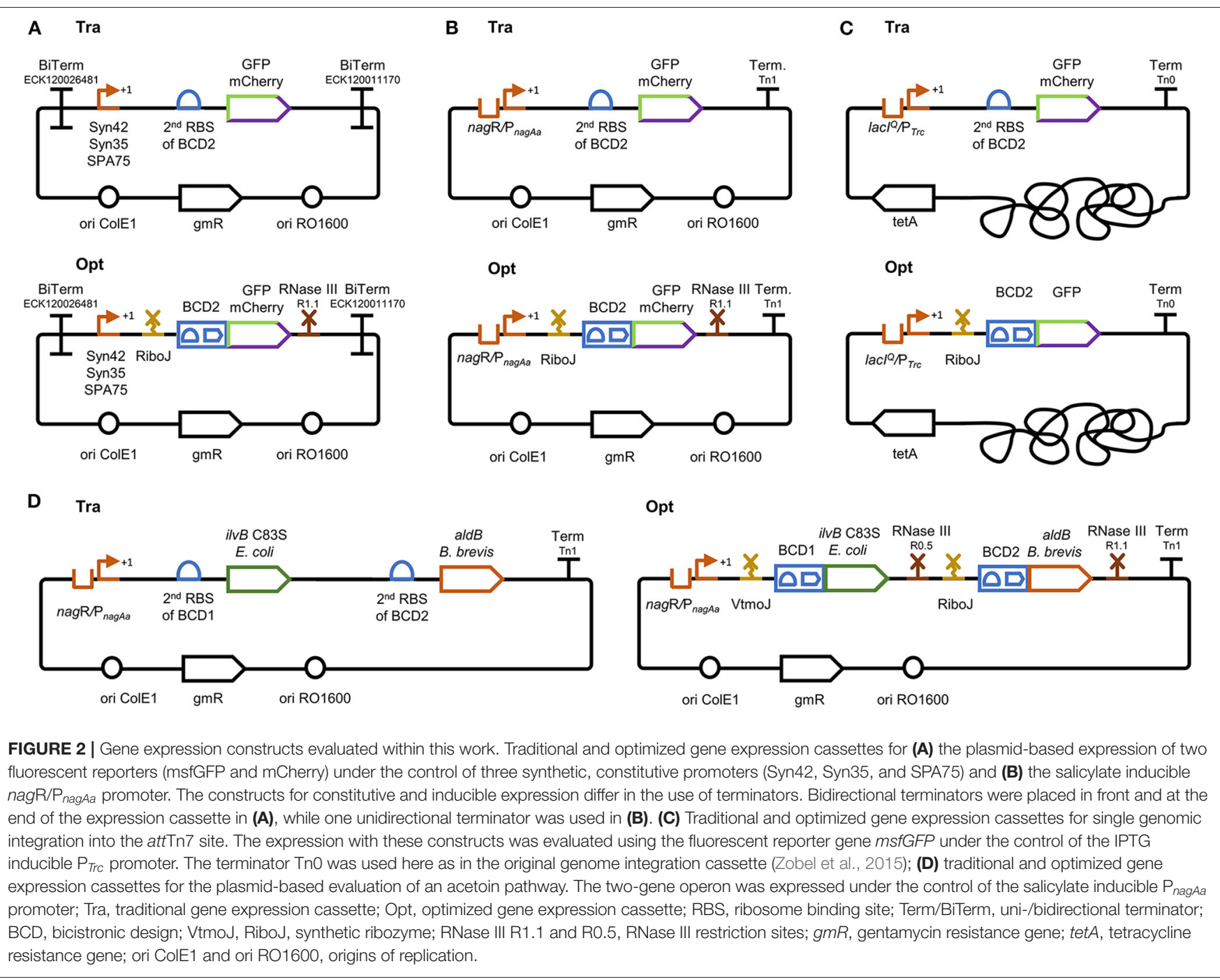

within the scientific community. Gene expression with the different constructs was characterized by the slope of the linear regression between measured fluorescence and cell dry weight, which indicates a specific expression strength.

All optimized gene expression constructs with the three tested constitutive promoters resulted in a substantial increase in fluorescence compared with their traditional counterparts (Figures 3A,B). While the ranking of the promoter strength was maintained with the optimized gene expression cassettes, absolute differences in the level of expression were reduced as a much higher increase was observed for the weaker Syn35 promoter expressing mCherry (Table 2). The lower fold increase in expression strength for the strong promoters suggests that the full potential of the optimized gene expression cassette in increasing protein expression is not reached here because of other cellular limitations, such as ribosome availability. This hypothesis is contrasted by a recent study, in which fluorescent protein expression in constructs harboring a RiboJ was evaluated using 24 different constitutive promoters covering a broad spectrum of expression strength (Clifton et al., 2018). From the presented single cell fluorescence values, a relatively stable fold change of the fluorescence output was observed for all tested constructs. Because of the distinct fluorescence measurements and standardization of the fluorescence values, the level of expression strength of the promoters used in the two studies cannot be compared. We can therefore not exclude that the SPA75 and Syn 42 promoters used in our study are significantly stronger than the strongest promoters used by Clifton et al. and that an inverse correlation between fold change improvement and basic promoter strength is only observed for promoters exerting a very high expression strength.

\section{Characterization of Plasmid-Based, Inducible Fluorescent Protein Expression}

Controlled gene expression is required, for instance, in genetic circuits or when the synthesis of a target product harms cell fitness and needs to be decoupled from growth (Terpe, 2006; Voigt, 2006; Chen, 2012; Jusiak et al., 2016). We, therefore, chose 

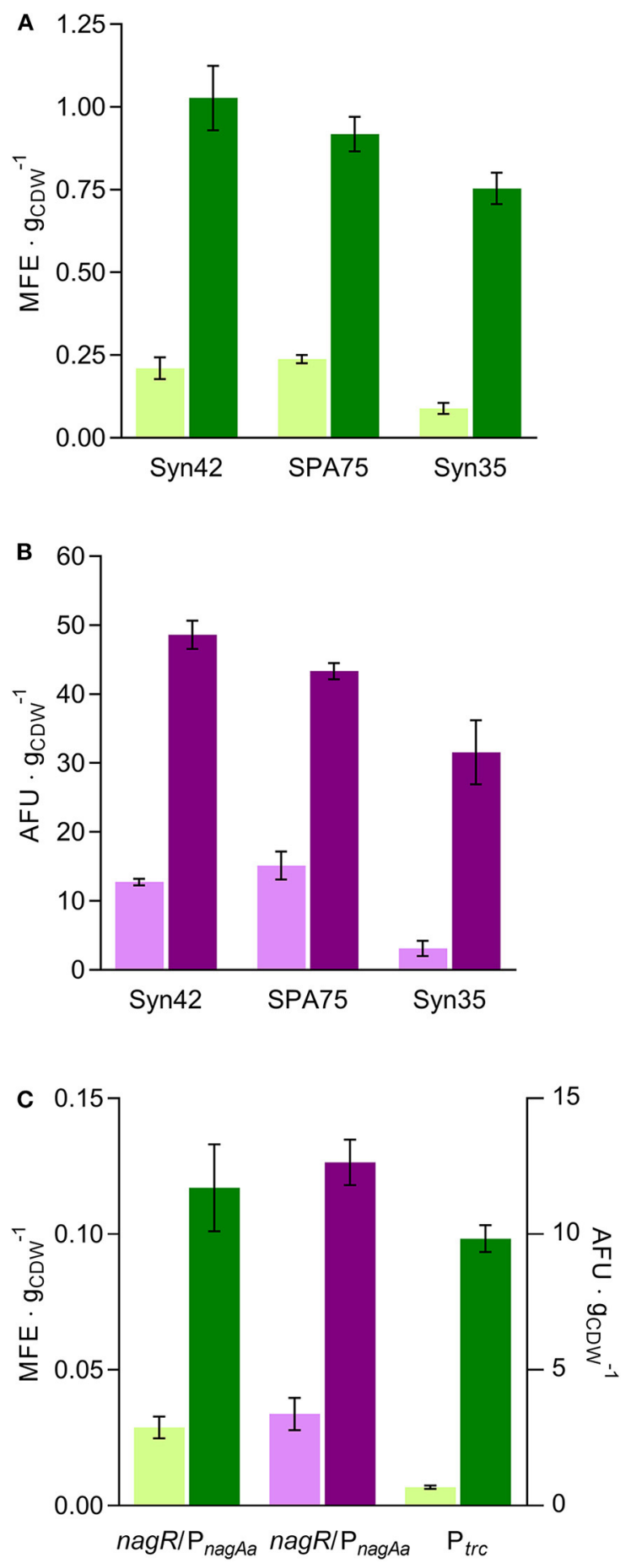

FIGURE 3 | Evaluation of the developed gene expression constructs: plasmid-based expression of msfGFP (green) and mCherry (pink) with the traditional (light-colored) or optimized (dark-colored) expression cassette employing (A/B) constitutive and (C, left and middle) the inducible PnagAa promoters; (C, right) msfGFP expression under the control of the inducible $\mathrm{P}_{\text {Trc }}$ promoter from single copies of the traditional and optimized gene expression cassettes integrated into the attTn7 site. Error bars indicate the standard deviation of three biological replicates except for the inducible nagR/P $P_{\text {nagAa }}$ construct expressing mCherry forwhich biological duplicates are represented. CDW, cell dry weight; MFE, $\mu$ moles of fluorescein equivalents; AFU, arbitrary fluorescence units.
TABLE 2 | Pairwise fold-changes of specific fluorescence (fluorescence per g cell dry weight) between the optimized and traditional gene expression cassettes calculated as described by Clifton et al. (2018).

\begin{tabular}{|c|c|c|c|c|c|}
\hline \multirow{2}{*}{$\begin{array}{l}\text { Expression } \\
\text { system } \\
\text { Promoter }\end{array}$} & \multicolumn{4}{|c|}{ pTN1 plasmid } & \multirow{2}{*}{$\begin{array}{l}\text {-attTn7:: } \\
\text { lac/ }{ }^{\mathrm{Q}} / \mathrm{P}_{T r c}\end{array}$} \\
\hline & Syn35 & Syn42 & SPA75 & $\begin{array}{c}\text { nagR/ } \\
\text { PnagAa }\end{array}$ & \\
\hline msfGFP & $8.7 \pm 1.6$ & $5.0 \pm 0.9$ & $3.9 \pm 0.3$ & $4.1 \pm 0.7$ & $14.7 \pm 1.6$ \\
\hline mCherry & $11.3 \pm 3.8$ & $3.8 \pm 0.2$ & $2.9 \pm 0.4$ & $3.9 \pm 0.7$ & - \\
\hline
\end{tabular}

to further evaluate inducible gene expression by placing the two fluorescent reporter proteins under control of the nagR $/ \mathrm{P}_{\text {nagAa }}$ promoter, inducible with low concentrations of the relatively cheap inducer salicylate (Hüsken et al., 2001). In both, the traditional and optimized expression constructs, the bidirectional terminators were replaced with the unidirectional terminator $\mathrm{Tn} 1$ as the first attempt to reduce the overall size of the cassette. The removal of the bidirectional terminators should bear similar effects in both cassettes as they have the identical genetic context. Aside from the removal of the bidirectional terminators and the use of a different promoter, both expression cassettes contained the equal genetic parts used for the evaluation of the constitutive promoters (Figure 2B).

Under the control of the inducible nagR/P $\mathrm{P}_{\text {nagAa }}$ promoter, the optimized gene expression cassette showed a similar behavior as with the strong constitutive promoters. The specific fluorescence was increased by 4 -fold with the optimized gene expression cassette in comparison to their respective traditional counterparts (Figure 3C). A similar increase was observed for the msfGFP signal under non-induced conditions whereas for the mCherry constructs only a 2-fold increase was observed (Noninduced data can be seen in Supplementary Figure 6). The stable increase of the msfGFP signal under both induced and uninduced conditions supports the concept of a post-transcriptional gene expression enhancement. The lower fold ratio in the noninduced conditions observed with the mCherry constructs could be related to analytical inaccuracies of the weaker mCherry signal and longer maturation time of this fluorescent protein in comparison to msfGFP. Inducible promoters, such as the nag $\mathrm{R} / \mathrm{P}_{\text {nagAa }}$ promoter system used in this work, tend to have a basal expression which could become problematic for the expression of toxic genes or their use in systems that need to be tightly regulated, e.g., genetic circuits. Attempts to achieve nonleaky inducible expression systems have been made, but their number continues to be limited (Horbal and Luzhetskyy, 2016). We propose the use of the optimized gene expression cassette in known non-leaky inducible promoter setups to increase the available expression range in such systems rather than aiming to engineer a novel non-leaky inducible variant. Since the additional parts of the optimized gene expression cassette tend to act on transcribed mRNA only, an optimized gene expression cassette harboring a tight, inducible promoter system could still exhibit the desired non-basal expression in the absence of the inducer and, once induced, reach higher expression levels than the standard counterpart. 
Characterization of Inducible Fluorescence Expression of Single, Genome-Integrated

\section{Constructs}

Genomic integration is generally preferred over plasmid-based expression when it comes to the stable construction of cell factories. Integrating the pathways into the genome grants higher genetic stability since it avoids common plasmidbased expression issues such as plasmid segregation and copy number variability (Jahn et al., 2014; Lindmeyer et al., 2015), plasmid replication-related growth impairment (Mi et al., 2016), and antibiotic dependency. However, genomic integration possesses specific disadvantages, like generally significantly lower expression levels and a limited number of characterized integration sites (Otto et al., 2019). A common approach to overcome the low expression levels of single genomic integration is to directly or randomly integrate the desired expression cassette at multiple sites. However, the directed multiple integration procedure is laborious and limited by the number of characterized integration sites, whereas random integration requires high-throughput screening. As we had seen a significant increase in expression strength with the optimized cassettes located on a plasmid, we argued that this device might also be valuable to boost the output of genome-integrated constructs. To evaluate if the optimized gene expression cassette could relief the low expression limitation of genomic integrations, single genomic integration cassettes expressing msfGFP were constructed and integrated at the neutral att $\mathrm{Tn} 7$ site.

While Cambray et al. (2013) have shown a very beneficial effect on standardized gene expression in constructs with the T7 bacteriophage derived R1.1 RNase III cleavage site, a negative effect on absolute expression levels cannot be ruled out. RNase III cleavage has been reported to have stabilizing as well as destabilizing effects depending on the mode of cleavage (Court, 1993; Laalami et al., 2014). While a single breakage, which leaves a folded structure at the $3^{\prime}$ end, stabilizes the processed mRNA, a double breakage that removes the hairpin induces enhanced degradation. The mode of cleavage at the R1.1. RNase III site from the T7 bacteriophage used in this study is contradictorily reported as harboring one or two cleavage sites and resulting in stabilized structures or mRNA molecules without $3^{\prime}$ secondary structures (Panayotatos and Truong, 1985; Calin-Jageman and Nicholson, 2003; Xiao et al., 2009). We left out the RNase III cleavage site in the genome-integrated optimized construct to avoid a potentially adverse effect on mRNA stability and hence protein abundance. Further expansion of the promoter scope was sought by utilizing the standard LacI-repressed $\mathrm{P}_{T r c}$ promoter for driving the gene expression of genome integrated cassettes. As in the plasmid-based evaluations, the optimized gene expression cassette harbored the RiboJ and BCD2, whereas the traditional version contained the $2^{\text {nd }}$ RBS of the BCD2 (Figure 2C).

Introducing this optimized gene expression cassette in the single genomic att $\operatorname{Tn} 7$ locus yielded a ca. 15-fold expression increase in comparison to the traditional analog, the highest fold increase observed in this work (Figure 3C). Even though
RNase III is not the major endoribonuclease responsible for mRNA turnover in bacteria, the enzyme does contribute to mRNA degradation (Deutscher, 2006). By removing the RNase III site from the optimized gene expression cassette, an mRNA degradation target was abolished, which might have resulted in an increased half-life of the transcripts and consequently higher expression levels.

The single, genome-integrated optimized expression cassette achieved expression levels of $0.098 \pm 0.005 \mu \mathrm{mol}$ fluorescein $\mathrm{g}^{-1}$ cell dry weight (CDW), an expression strength in the range of the plasmid-based optimized cassette under the control of the inducible promoter $n a g R / \mathrm{P}_{n a g A a}$ or the constitutive promoter Syn35 within the traditional cassette. Although the use of different promoters does not allow a fair comparison, it is still noteworthy to state that expression levels were achieved with the single genomic integration cassette, which are usually seen for episomally expressed genes.

\section{Evaluation of a Recombinant Acetoin Production Pathway Employing the Optimized Expression Cassette}

Finally, a heterologous acetoin pathway was cloned within the pTN1 plasmid backbone to further evaluate the optimized expression cassette in a production context. The acetoin pathway included a C83S mutant of the E. coli K-12 MG1655 acetolactate synthase $(i l v B)$ and an acetolactate decarboxylase (aldB) from Brevibacillus brevis (Figure 4A). The E. coli ilvB C83S mutant was chosen due to its 4 -fold lower $\mathrm{K}_{\mathrm{m}}$ and $126 \%$ higher $\mathrm{k}_{\mathrm{cat}} / \mathrm{K}_{\mathrm{m}}$ ratio compared with the wild type, making it one of the most efficient enzymes of this class so far characterized (Belenky et al., 2012). The B. brevis aldB was selected based on its
A

A
2<smiles>CC(=O)C(=O)OC(C)C(=O)O</smiles>
lactate $\mathrm{aldB} \leadsto \mathrm{CO}_{2}$

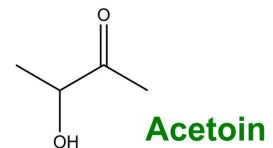<smiles>CC(=O)CC(O)C(=O)OCc1ccccc1</smiles>

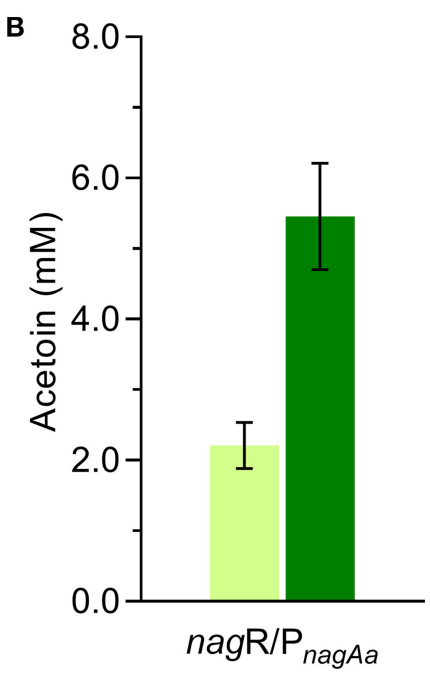

FIGURE 4 | Acetoin production pathway comprising the C83S ilvB mutant from E. coli and aldB from Brevibacillus brevis (A); acetoin titers achieved by the plasmid-based expression of the 2-gene operon under the control of the inducible $\mathrm{P}_{\text {nagAa }}$ promoter employing a traditional and an optimized expression cassette (B) 
low $\mathrm{K}_{\mathrm{m}}$ value (Rostgaard et al., 1987). In the polycistronic design, expression of both genes was driven by the inducible nag $\mathrm{R} / \mathrm{P}_{\text {nagAa }}$ promoter system while each gene was framed with a ribozyme, a bicistronic design version, and an RNase III site. The acetolactate synthase was framed with the VtmoJ ribozyme gene, the BCD1 bicistronic design, and the RNase III R0.5 site, whereas the acetolactate decarboxylase was surrounded with the RiboJ ribozyme gene, the BCD2 bicistronic design, and the RNase III R1.1 site (Figure 2D). The traditional expression cassette was obtained by removing the expression enhancing genetic parts while maintaining the $2^{\text {nd }}$ RBS of BCD1 upstream of the $i l v B$ and the $2^{\text {nd }}$ RBS of BCD2 upstream of the aldB (Figure 2D).

Employing the optimized gene expression architecture to the acetoin pathway led to an acetoin accumulation of 5.5 $\pm 0.76 \mathrm{mM}$, representing a 2.5 -fold production increase when compared to the traditional counterpart (Figure 4B). The increase of acetoin production solely using the optimized gene expression cassette shows that its use can be extended to production contexts. The optimized gene expression cassette could be advantageous for metabolic engineering approaches where high gene expression is required, such as redirecting native metabolites to the desired production pathway or for enzyme production for in vitro applications.

\section{qPCR Based Elucidation of mRNA Stability}

The high fluorescence expression levels and increased acetoin production achieved by the optimized gene expression in this work arose from the combination of mRNA stabilizing and translation boosting genetic parts. Lou et al. integrated hairpins in both ribozymes used in this work, RiboJ and VtmoJ, to expose the RBS and confirmed their catalytic functionality through rapid amplification of cDNA $5^{\prime}$-ends (Lou et al., 2012). The presence of such hairpins in the $5^{\prime} \mathrm{UTR}$ region was reported to increase the respective mRNA half-life, which leads to higher protein production (Viegas et al., 2018). Further clarification of the stabilizing effect of the hairpin structure of RiboJ and its individual effect on protein expression has been published elsewhere (Clifton et al., 2018). To evaluate if such a phenomenon occurred in the optimized gene expression cassette and contributed to the lump sum of increased protein expression, qPCR assays were performed to compare the decay rates of the transcripts. For this purpose, cultivations of the strains expressing msfGFP under the control of the SPA75 promoter were treated with rifampicin and nalidixic acid in the early exponential growth phase, and mRNA samples were retrieved over time (see Supplementary Figure 7). The addition of rifampicin inhibits bacterial RNA polymerase, whereas nalidixic acid inhibits a subunit of the DNA gyrase and topoisomerase. As the mRNA abundance data of the msfGFP were normalized with the data of the housekeeping gene $r p o B$ to cancel out small differences in the template concentration, the determination of the absolute decay rate of the msfGFP transcript was not possible. Instead, we calculated the difference between the decay rates of the $r p o B$ and the msfGFP mRNA (i.e., $k_{r p o B}-k_{m s f G F P}$, with $k$ being the decay rate). Given that the $r p o B$ mRNA decay should be equal in both strains the delta can be used to reveal a possible

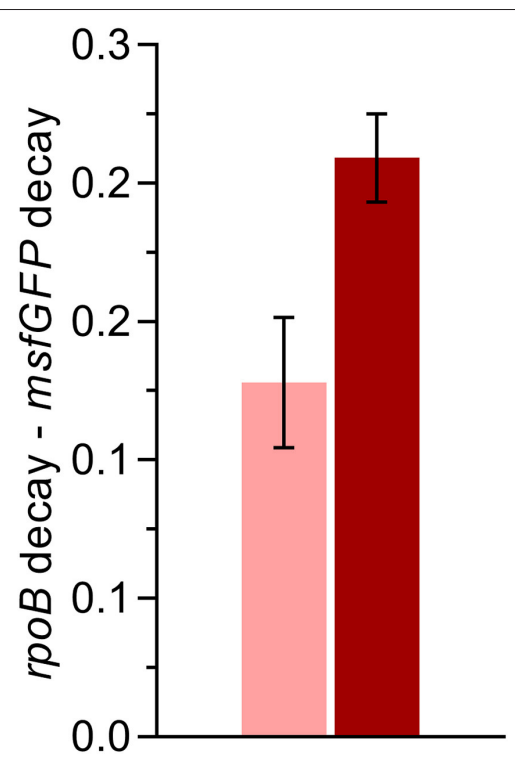

FIGURE 5 | qPCR based elucidation of mRNA stability of msfGFP transcripts from the plasmid-based traditional (light-colored) and optimized (dark-colored) expression cassettes harboring the constitutive SPA75 promoter. Early exponential cultivations were treated with the antibiotics, nalidixic acid and rifampicin, to halt DNA replication and transcription, respectively. Samples were taken over time (Time course data are shown in Supplementary Figure 7), and mRNA levels were assessed through qPCR. mRNA decay rates of transcripts from each expression cassette were retrieved through the difference between the decay rate of the housekeeping gene rpo $B$ and the target msfGFP. Error bars indicate the standard deviation of two biological replicates.

difference in the $m s f G F P$ decay rates in the optimized and traditional expression architecture. As hypothesized, the data was significantly higher for the optimized construct (1.6-fold), which translates to a reduced decay rate of the msfGFP transcript (Figure 5). Hence, higher mRNA stability is indeed one factor contributing to the observed increase in fluorescence output and acetoin production.

\section{CONCLUSION}

In this study, the optimized gene expression cassette architecture proposed by Nielsen et al. was evaluated in P. taiwanensis VLB120 for the expression of fluorescent reporter genes and a 2-step acetoin biosynthesis operon (Nielsen et al., 2013). The optimized gene expression cassette was characterized on a plasmid or single genomic integration basis with either constitutive or inducible promoters to cover all commonly used expression approaches in metabolic engineering. In all evaluations, the optimized gene expression cassette outperformed its traditional counterpart. The highest fold improvement was observed once the RNase III site was removed and evaluated on a single genomic integration basis under the control of the IPTG inducible $\mathrm{P}_{\text {Trc }}$ promoter. Such a boost allowed a single genomic integration-based expression to achieve expression levels commonly reached with plasmids. Within the constitutive promoter paradigm, the optimized gene expression cassette 
increased expression levels of the strongest promoter of a promoter library, showing that this tool could be used to extend expression ranges further. The mRNA transcripts retrieved by the optimized gene expression cassette harnessed higher stability than the transcripts from the traditional counterpart, validating that mRNA stability contributed to the observed results. This work demonstrates the applicability of the optimized gene expression cassette as a tool to achieve high gene expression levels through transcriptionindependent approaches that rely on mRNA stability and translation efficiency.

\section{DATA AVAILABILITY STATEMENT}

The raw data supporting the conclusions of this article will be made available by the authors, without undue reservation, to any qualified researcher.

Primers, annotated sequences of ordered DNA fragments, conversion of Biolector fluorescence a.u. into $\mu \mathrm{M}$ fluorescein and qPCR primer pair efficiencies, fluorescence evaluation of the inducible gene expression constructs in induced and uninduced cultures, and time course data of normalized msfGFP mRNA abundance are provided in the Supplementary Material.

\section{REFERENCES}

Beal, J., Haddock-Angelli, T., Baldwin, G., Gershater, M., Dwijayanti, A., Storch, M., et al. (2018). Quantification of bacterial fluorescence using independent calibrants. PLoS ONE 13:e0199432. doi: 10.1371/journal.pone.0199432

Belenky, I., Steinmetz, A., Vyazmensky, M., Barak, Z., Tittmann, K., and Chipman, D. M. (2012). Many of the functional differences between acetohydroxyacid synthase (AHAS) isozyme I and other AHASs are a result of the rapid formation and breakdown of the covalent acetolactate-thiamin diphosphate adduct in AHAS I. FEBS J. 279, 1967-1979. doi: 10.1111/j.1742-4658.2012.0 8577.x

Borkowski, O., Ceroni, F., Stan, G. B., and Ellis, T. (2016). Overloaded and stressed: whole-cell considerations for bacterial synthetic biology. Curr. Opin. Microbiol. 33, 123-130. doi: 10.1016/j.mib.2016.07.009

Calin-Jageman, I., and Nicholson, A. W. (2003). Mutational analysis of an RNA internal loop as a reactivity epitope for Escherichia coli ribonuclease III substrates. Biochemistry 42, 5025-5034. doi: 10.1021/bi0 $30004 \mathrm{r}$

Cambray, G., Guimaraes, J. C., Mutalik, V. K., Lam, C., Mai, Q. A., Thimmaiah, T., et al. (2013). Measurement and modeling of intrinsic transcription terminators. Nucleic Acids Res. 41, 5139-5148. doi: 10.1093/nar/ gkt163

Carneiro, S., Ferreira, E. C., and Rocha, I. (2013). Metabolic responses to recombinant bioprocesses in Escherichia coli. J. Biotechnol. 164, 396-408. doi: 10.1016/j.jbiotec.2012.08.026

Carrier, T. A., and Keasling, J. D. (1997). Engineering mRNA stability in E. coli by the addition of synthetic hairpins using a $5^{\prime}$ cassette system. Biotechnol. Bioeng. $55,577-580$.

Chen, R. (2012). Bacterial expression systems for recombinant protein production: E. coli and beyond. Biotechnol. Adv. 30, 1102-1107. doi: 10.1016/j.biotechadv.2011.09.013

Chen, Y. J., Liu, P., Nielsen, A. A., Brophy, J. A., Clancy, K., Peterson, T., et al. (2013). Characterization of 582 natural and synthetic terminators and quantification of their design constraints. Nat. Methods 10, 659-664. doi: $10.1038 /$ nmeth. 2515

\section{AUTHOR CONTRIBUTIONS}

DN and BE conceived the study with the help of LB. BE and LB supervised the study. DN performed all experiments with the support of SV. All authors analyzed the data and approved the final version of the manuscript.

\section{FUNDING}

This study has been conducted within the ERA SynBio project SynPath (Grant ID 031A459) with the financial support of the German Federal Ministry of Education and Research.

\section{ACKNOWLEDGMENTS}

We thank Maike Otto and Nick Wierckx for useful discussions. We are grateful to Ethan M. Jones, Kalen P. Clifton, and Margaret S. Saha for valuable input and discussion.

\section{SUPPLEMENTARY MATERIAL}

The Supplementary Material for this article can be found online at: https://www.frontiersin.org/articles/10.3389/fbioe. 2019.00458/full\#supplementary-material

Chomczynski, P., and Rymaszewski, M. (2006). Alkaline polyethylene glycol-based method for direct PCR from bacteria, eukaryotic tissue samples, and whole blood. Biotechniques 40, 454-458. doi: 10.2144/000112149

Clifton, K. P., Jones, E. M., Paudel, S., Marken, J. P., Monette, C. E., Halleran, A. D., et al. (2018). The genetic insulator RiboJ increases expression of insulated genes. J. Biol. Eng. 12:23. doi: 10.1186/s13036-018-0115-6

Court, D. (1993). “5 - RNA processing and degradation by RNase III," in Control of Messenger RNA Stability, eds J. G. Belasco and G. Brawerman (San Diego, CA: Academic Press), 71-116. doi: 10.1016/B978-0-08-091652-1.50009-8

Davy, A. M., Kildegaard, H. F., and Andersen, M. R. (2017). Cell factory engineering. Cell Syst. 4, 262-275. doi: 10.1016/j.cels.2017.02.010

Deutscher, M. P. (2006). Degradation of RNA in bacteria: comparison of mRNA and stable RNA. Nucleic Acids Res. 34, 659-666. doi: 10.1093/nar/gkj472

Ditta, G., Stanfield, S., Corbin, D., and Helinski, D. R. (1980). Broad host range DNA cloning system for gram-negative bacteria: construction of a gene bank of Rhizobium meliloti. Proc. Natl. Acad. Sci. U.S.A. 77, 7347-7351. doi: 10.1073/pnas.77.12.7347

Gibson, D. G., Young, L., Chuang, R. Y., Venter, J. C., Hutchison, C. A. 3rd, and Smith, H. O. (2009). Enzymatic assembly of DNA molecules up to several hundred kilobases. Nat. Methods 6, 343-345. doi: 10.1038/nmeth.1318

Hanahan, D. (1985). Techniques for transformation of E. coli. DNA cloning: a practical approach. 1, 109-135.

Horbal, L., and Luzhetskyy, A. (2016). Dual control system - a novel scaffolding architecture of an inducible regulatory device for the precise regulation of gene expression. Metab. Eng. 37, 11-23. doi: 10.1016/j.ymben.2016.03.008

Hüsken, L. E., Beeftink, R., de Bont, J. A., and Wery, J. (2001). Highrate 3-methylcatechol production in Pseudomonas putida strains by means of a novel expression system. Appl. Microbiol. Biotechnol. 55, 571-577. doi: 10.1007/s002530000566

Jahn, M., Vorpahl, C., Turkowsky, D., Lindmeyer, M., Buhler, B., Harms, H., et al. (2014). Accurate determination of plasmid copy number of flow-sorted cells using droplet digital PCR. Anal. Chem. 86, 5969-5976. doi: 10.1021/ac501118v

Jusiak, B., Cleto, S., Perez-Pinera, P., and Lu, T. K. (2016). Engineering synthetic gene circuits in living cells with CRISPR technology. Trends Biotechnol. 34, 535-547. doi: 10.1016/j.tibtech.2015.12.014 
Köhler, K. A., Rückert, C., Schatschneider, S., Vorholter, F. J., Szczepanowski, R., Blank, L. M., et al. (2013). Complete genome sequence of Pseudomonas sp. strain VLB120 a solvent tolerant, styrene degrading bacterium, isolated from forest soil. J. Biotechnol. 168, 729-730. doi: 10.1016/j.jbiotec.2013.10.016

Laalami, S., Zig, L., and Putzer, H. (2014). Initiation of mRNA decay in bacteria. Cell. Mol. Life Sci. 71, 1799-1828. doi: 10.1007/s00018-013-1472-4

Landgraf, D. (2012). Quantifying localizations and dynamics in single bacterial cells (Doctoral dissertation). Harvard University.

Lang, K., Zierow, J., Buehler, K., and Schmid, A. (2014). Metabolic engineering of Pseudomonas sp. strain VLB120 as platform biocatalyst for the production of isobutyric acid and other secondary metabolites. Microb. Cell Fact. 13:2. doi: 10.1186/1475-2859-13-2

Lenzen, C., Wynands, B., Otto, M., Bolzenius, J., Mennicken, P., Blank, L. M., et al. (2019). High-yield production of 4-hydroxybenzoate from glucose or glycerol by an engineered Pseudomonas taiwanensis VLB120. Front. Bioeng. Biotechnol. 7:130. doi: 10.3389/fbioe.2019.00130

Lindmeyer, M., Jahn, M., Vorpahl, C., Muller, S., Schmid, A., and Bühler, B. (2015). Variability in subpopulation formation propagates into biocatalytic variability of engineered Pseudomonas putida strains. Front. Microbiol. 6:1042. doi: 10.3389/fmicb.2015.01042

Liu, L., Yang, H., Shin, H. D., Chen, R. R., Li, J., Du, G., et al. (2013). How to achieve high-level expression of microbial enzymes: strategies and perspectives. Bioengineered 4, 212-223. doi: 10.4161/bioe.24761

Lou, C., Stanton, B., Chen, Y. J., Munsky, B., and Voigt, C. A. (2012). Ribozymebased insulator parts buffer synthetic circuits from genetic context. Nat. Biotechnol. 30, 1137-1142. doi: 10.1038/nbt.2401

Martinez-Garcia, E., and de Lorenzo, V. (2011). Engineering multiple genomic deletions in Gram-negative bacteria: analysis of the multi-resistant antibiotic profile of Pseudomonas putida KT2440. Environ. Microbiol. 13, 2702-2716. doi: 10.1111/j.1462-2920.2011.02538.x

Mi, J., Sydow, A., Schempp, F., Becher, D., Schewe, H., Schrader, J., et al. (2016). Investigation of plasmid-induced growth defect in Pseudomonas putida. J. Biotechnol. 231, 167-173. doi: 10.1016/j.jbiotec.2016.06.001

Mutalik, V. K., Guimaraes, J. C., Cambray, G., Lam, C., Christoffersen, M. J., Mai, Q. A., et al. (2013). Precise and reliable gene expression via standard transcription and translation initiation elements. Nat. Methods 10, 354-360. doi: $10.1038 /$ nmeth. 2404

Nielsen, A. A., Der, B. S., Shin, J., Vaidyanathan, P., Paralanov, V., Strychalski, E. A., et al. (2016). Genetic circuit design automation. Science 352:aac7341. doi: $10.1126 /$ science.aac7341

Nielsen, A. A., Segall-Shapiro, T. H., and Voigt, C. A. (2013). Advances in genetic circuit design: novel biochemistries, deep part mining, and precision gene expression. Curr. Opin. Chem. Biol. 17, 878-892. doi: 10.1016/j.cbpa.2013.10.003

Otto, M., Wynands, B., Drepper, T., Jaeger, K. E., Thies, S., Loeschcke, A., et al. (2019). Targeting 16S rDNA for stable recombinant gene expression in Pseudomonas. ACS Synth. Biol. 8, 1901-1912. doi: 10.1021/acssynbio.9b00195

Panayotatos, N., and Truong, K. (1985). Cleavage within an RNase III site can control mRNA stability and protein synthesis in vivo. Nucleic Acids Res. 13, 2227-2240. doi: 10.1093/nar/13.7.2227

Panke, S., Witholt, B., Schmid, A., and Wubbolts, M. G. (1998). Towards a biocatalyst for $(S)$-styrene oxide production: characterization of the styrene degradation pathway of Pseudomonas sp. strain VLB120. Appl. Environ. Microbiol. 64, 2032-2043. doi: 10.1128/AEM.64.6.2032-2043.1998

Puigbò, P., Guzmán, E., Romeu, A., and Garcia-Vallvé, S. (2007). OPTIMIZER: a web server for optimizing the codon usage of DNA sequences. Nucleic Acids Res. 35, W126-W131. doi: 10.1093/nar/gkm219
Rostgaard, J. B., Svendsen, I., and Oitese, M. (1987). Isolation and characterization of an alpha-acetolactate decarboxylase useful for accelerated beer maturation. Proc. Congr. Eur. Brew. Conv. 93, 160-160.

Salis, H. M., Mirsky, E. A., and Voigt, C. A. (2009). Automated design of synthetic ribosome binding sites to control protein expression. Nat. Biotechnol. 27, 946-950. doi: 10.1038/nbt.1568

Schmitz, S., Nies, S., Wierckx, N., Blank, L. M., and Rosenbaum, M. A. (2015). Engineering mediator-based electroactivity in the obligate aerobic bacterium Pseudomonas putida KT2440. Front. Microbiol. 6:284. doi: 10.3389/fmicb.2015.00284

Terpe, K. (2006). Overview of bacterial expression systems for heterologous protein production: from molecular and biochemical fundamentals to commercial systems. Appl. Microbiol. Biotechnol. 72, 211-222. doi: 10.1007/s00253-00 6-0465-8

Verhoef, S., Wierckx, N., Westerhof, R. G., de Winde, J. H., and Ruijssenaars, H. J. (2009). Bioproduction of p-hydroxystyrene from glucose by the solventtolerant bacterium Pseudomonas putida S12 in a two-phase water-decanol fermentation. Appl. Environ. Microbiol. 75, 931-936. doi: 10.1128/AEM.0 2186-08

Viegas, S. C., Apura, P., Martínez-García, E., de Lorenzo, V., and Arraiano, C. M. (2018). Modulating heterologous gene expression with portable mRNA-stabilizing 5'-UTR sequences. ACS Synth. Biol. 7, 2177-2188. doi: 10.1021/acssynbio.8b00191

Voigt, C. A. (2006). Genetic parts to program bacteria. Curr. Opin. Biotechnol. 17, 548-557. doi: 10.1016/j.copbio.2006. 09.001

Wierckx, N. J., Ballerstedt, H., de Bont, J. A., and Wery, J. (2005). Engineering of solvent-tolerant Pseudomonas putida S12 for bioproduction of phenol from glucose. Appl. Environ. Microbiol. 71, 8221-8227. doi: 10.1128/AEM.71.12.8221-82 27.2005

Wynands, B., Lenzen, C., Otto, M., Koch, F., Blank, L. M., and Wierckx, N. (2018). Metabolic engineering of Pseudomonas taiwanensis VLB120 with minimal genomic modifications for high-yield phenol production. Metab. Eng. 47, 121-133. doi: 10.1016/j.ymben.2018. 03.011

Xiao, J., Feehery, C. E., Tzertzinis, G., and Maina, C. V. (2009). E. coli RNase III(E38A) generates discrete-sized products from long dsRNA. RNA 15, 984-991. doi: 10.1261/rna.1196509

Zobel, S., Benedetti, I., Eisenbach, L., de Lorenzo, V., Wierckx, N., and Blank, L. M. (2015). Tn7-based device for calibrated heterologous gene expression in Pseudomonas putida. ACS Synth. Biol. 4, 1341-1351. doi: 10.1021/acssynbio.5b 00058

Conflict of Interest: The authors declare that the research was conducted in the absence of any commercial or financial relationships that could be construed as a potential conflict of interest.

Copyright (c) 2020 Neves, Vos, Blank and Ebert. This is an open-access article distributed under the terms of the Creative Commons Attribution License (CC BY). The use, distribution or reproduction in other forums is permitted, provided the original author(s) and the copyright owner(s) are credited and that the original publication in this journal is cited, in accordance with accepted academic practice. No use, distribution or reproduction is permitted which does not comply with these terms. 